\title{
Pharmaceutical contamination in residential, industrial, and agricultural waste streams: Risk to aqueous environments in Taiwan
}

\author{
Angela Yu-Chen Lin *, Tsung-Hsien Yu, Cheng-Fang Lin \\ National Taiwan University, Graduate Institute of Environmental Engineering, No. 1, Section 4, Roosevelt Road, Taipei 10617, Taiwan
}

\section{A R T I C L E I N F O}

\section{Article history:}

Received 16 June 2008

Received in revised form 11 August 2008

Accepted 14 August 2008

Available online 30 September 2008

\section{Keywords:}

Pharmaceuticals

Antibiotics

Wastewaters

Hospitals

Aquacultures

Ecotoxicity

\begin{abstract}
A B S T R A C T
This is a comprehensive study of the occurrence of antibiotics, hormones and other pharmaceuticals in water sites that have major potential for downstream environmental contamination. These include residential (hospitals, sewage treatment plants, and regional discharges), industrial (pharmaceutical production facilities), and agricultural (animal husbandries and aquacultures) waste streams. We assayed 23 Taiwanese water sites for 97 targeted compounds, of which a significant number were detected and quantified. The most frequently detected compounds were sulfamethoxazole, caffeine, acetaminophen, and ibuprofen, followed closely by cephalexin, ofloxacin, and diclofenac, which were detected in $>91 \%$ of samples and found to have median (maximum) concentrations of 0.2 (5.8), 0.39 (24.0), 0.02 (100.4), 0.41 (14.5), 0.15 (31.4), 0.14 (13.6) and $0.083(29.8) \mu \mathrm{g} / \mathrm{L}$, respectively. Lincomycin and acetaminophen had high measured concentrations $(>100 \mu \mathrm{g} / \mathrm{L})$, and 35 other pharmaceuticals occurred at the $\mu \mathrm{g} / \mathrm{L}$ level. These incidence and concentration results correlate well with published data for other worldwide locations, as well as with Taiwanese medication usage data, suggesting a human contamination source. Many pharmaceuticals also occurred at levels exceeding predicted no-effect concentrations (PNEC), warranting further investigation of their occurrence and fate in receiving waters, as well as the overall risks they pose for local ecosystems and human residents. The information provided here will also be useful for development of strategies for regulation and remediation.
\end{abstract}

(c) 2008 Elsevier Ltd. All rights reserved.

\section{Introduction}

In recent years, a significant body of work has identified trace pharmaceuticals in natural aquatic environments. Some degraded naturally through hydrolysis (Kuehne et al., 2000), biodegradation (Morrall et al., 2004; Lin et al., 2006), or direct and indirect photolysis (Tixier et al., 2002; Quanrud et al., 2004; Lin and Reinhard, 2005; Lin et al., 2006), while others had the potential to be sorbed and eliminated from aqueous environments (Morrall et al., 2004; Sassman and Lee, 2005), but many compounds persisted in aqueous systems. Ubiquitous occurrences of these compounds (antibiotics, hormones, non-steroidal anti-inflammatory drugs (NSAIDs), lipid regulators, $\beta$-blockers, psychiatric drugs, etc.) have been reported for rivers, lakes, and reservoirs around the world (Golet et al., 2002; Kolpin et al., 2002; Gross et al., 2004; Brown et al., 2006; Lin et al., 2006; Kim et al., 2007). For example, Heberer identified ng/L levels of clofibric acid in tap water from Berlin, Germany (Heberer, 2002), while other studies detected significant and measurable concentrations of ibuprofen, diclofenac, and carbamazepine in finished drinking waters (Ternes et al., 2002; Loraine and Pettigrove, 2006; Kim et al., 2007).

\footnotetext{
* Corresponding author. Tel.: +886 23366 3729; fax: +8862 23928830 .

E-mail address: yuchenlin@ntu.edu.tw (A.Y.-C. Lin).
}

Waste streams from hospitals, wastewater treatment plants (WWTPS), and sewage treatment plants (STPs) have been identified as major contributors to environmental contamination with human-derived medications (Kummerer and Henninger, 2003; Gross et al., 2004; Miao et al., 2004; Brown et al., 2006; Gomez et al., 2006; Kim et al., 2007; Xu et al., 2007). Few studies have attempted to correlate the use of veterinary medicines in areas supporting dairy and agricultural industries with occurrence of these compounds in local waters. Brown et al. (2006) identified $\mu \mathrm{g} / \mathrm{L}$ levels of lincomycin in two dairies studied in New Mexico. Managaki et al. (2007) found macrolide and sulfonamide antibiotics at ng/L levels in Vietnam's Mekong Delta and compared them with levels in the Tamagawa River in Japan. However, the existing information on contamination sources is spotty and limited, and normally several methods are needed to identify multiple pharmaceutical classes which are present only at trace levels (ng/L).

Taiwan is a densely populated island, with a population of 22.77 million people residing in a mere $36188 \mathrm{~km}^{2}$. The island has 19433 hospitals, whose total drug consumption for the year 2005 is estimated at 13.2 billion doses (Directorate-General of Budget, 2008; National Research Health Research Database, 2008). Hospital effluents and regional discharges can be important sources of pharmaceutical contaminations, especially in urban regions. In rural areas, however, agriculture, animal husbandry, and aquaculture are an 
important segment of the economy, and waste from these sectors represents an additional potential source of contamination with veterinary medicines such as antibiotics and estrogens.

Our target analytes include human and veterinary medicines from ten antibiotic classes (sulfonamides, tetracyclines, lincosamides, macrolides, penicillins, cephalosporins, imidazoles, quinolones, chloramphenicols and others), and from estrogens, $\beta$-blockers, psychostimulants, vasodilators, psychiatric agents, NSAIDs, and lipid-regulator/cholesterol-lowering medication groups. These compounds were chosen for their high detection frequencies, as reported by investigators worldwide, and for their significant usage by the global population and by Taiwanese especially. Samples were taken at important water sites that are susceptible to pharmaceutical contaminations and that have the potential to become contamination sources in themselves. Because it would be impractical to attempt individual analyses for each of our 97 target drugs on samples from $>20$ sites, we used an analytical method to measure a sweep of all 97 pharmaceuticals simultaneously, even if present at only trace levels (low ng/L). Aquatic toxicity results were integrated with local medicine usage data and compared for potential environmental and human risk.

\section{Experiment}

\subsection{Site selection and sampling}

Limited data are available on the environmental occurrence in Taiwan of most of our targeted pharmaceuticals. As a consequence, we selected sampling sites from areas/places that were likely to be contaminated with human (hospitals, STPs, regional discharges), industrial (pharmaceutical production facilities), or agricultural (animal husbandry and aquaculture) wastewaters, and that were likely to pass their pharmaceutical load to downstream environmental waters. Regional discharges were the contents of sizeable sewage pipes collecting mainly household wastewaters and rainwater before being released to rivers and oceans. Triplicate grab samples were collected from 23 selected sites (waste streams and effluents from three pharmaceutical production facilities, six hospitals, five STPs, five regional discharges, two animal husbandries, and three aquacultures) in one-liter amber glass bottles and stored in ice-packed coolers. Detailed descriptions of each sampling site are provided in the Supplementary material section. Briefly, waste stream samples were collected at the exit points (final effluent) of each facility with or without being treated. Wastewater effluents from hospitals and pharmaceutical production facilities were only treated to meet minimal effluent standards: BOD $30 \mathrm{mg} / \mathrm{L}$, COD $100 \mathrm{mg} / \mathrm{L}, \mathrm{S} . S-30 \mathrm{mg} / \mathrm{L}$, true color 550, E. coli $200000 \mathrm{CFU} / 100 \mathrm{~mL}$. STPs were equipped with secondary treatment units, while agricultural wastewaters were not treated before releasing to downstream rivers and oceans. Eight $\mathrm{mL}$ of $0.125 \mathrm{M}$ EDTA-2Na were added to sampling bottles prior to sample collection. All samples were collected from Jan. 15-31st, 2008, and analysis was completed within two weeks of sample collection.

\subsection{Analytical methods}

The following method was developed to identify and quantify up to 97 targeted pharmaceuticals simultaneously.

\subsubsection{Sample preparation}

All water and wastewater samples were vacuum-filtered through a $0.45 \mu \mathrm{m}$ and $0.22 \mu \mathrm{m}$ cellulose acetate membrane filter, acidified to $\mathrm{pH} 4.0$ using sulfuric acid $(2 \mathrm{~N})$, and stored at $4{ }^{\circ} \mathrm{C}$ until analysis. Oasis HLB cartridges (500 mg, $6 \mathrm{~mL}$, Waters, Milford, MA, USA) used for solid phase extraction (SPE) were preconditioned with $6 \mathrm{~mL}$ of methanol and $6 \mathrm{~mL}$ of deionized (DI) water. Aliquots of $400 \mathrm{~mL}$ water samples were spiked with ${ }^{13} \mathrm{C}_{6}$-sulfamethazine (employed as a surrogate) and loaded to the cartridges with a flow rate of 3-6 $\mathrm{mL} / \mathrm{min}$. After sample passage, cartridges were rinsed with $6 \mathrm{~mL}$ DI water to remove excess EDTA-2Na and dried with a flow of nitrogen gas. After drying, analytes were eluted with $4 \mathrm{~mL}$ of methanol and $4 \mathrm{~mL}$ of methanol-diethylether (50:50, v/ v). The eluates were collected, evaporated to dryness with nitrogen stream, and reconstituted to $0.4 \mathrm{~mL}$ with $25 \%$ aqueous methanol. Final solutions were filtered through a $0.45 \mu \mathrm{m}$ PVDF membrane filter before liquid chromatography/tandem mass spectrometry (HPLC-MS/MS) analysis.

\subsubsection{LC-ESI-tandem MS analysis}

Chromatographic separation of analytes was performed using an Agilent 1200 module (Agilent Technologies, Palo Alto, CA, USA) equipped with a Phenomenex Luna C18 column $(150 \times 4.6 \mathrm{~mm}, 5 \mu \mathrm{m})$. A binary gradient with a flow rate of $1.0 \mathrm{~mL} / \mathrm{min}$ was used. Mobile phase A contained $0.1 \%$ formic acid $(\mathrm{v} / \mathrm{v})$ in water. Mobile phase B contained $0.1 \%$ formic acid (v/v) in methanol. The gradient started with $0 \%$ of mobile phase $B$ for $0.5 \mathrm{~min}$, increased to $40 \%$ from $0.5-3 \mathrm{~min}$, to $70 \%$ from 3.0 $7.5 \mathrm{~min}$, to $95 \%$ from $7.5-9.0 \mathrm{~min}$, remained at $95 \%$ until $11 \mathrm{~min}$, decreased to $0 \%$ from $11-12 \mathrm{~min}$, and remained at $0 \%$. All target compounds were eluted out of the column within 15 min. The sample injection volume was $50 \mu \mathrm{L}$. The autosampler was operated at room temperature.

The mass spectrometric measurements were carried out on a Sciex API 4000 (Applied Biosystems, Foster City, CA, USA) equipped with a turbo ionspray source. Analyses were performed in negative mode for 18 compounds (all NSAIDs except for acetaminophen; mepirizole and famotidine; all chloramphenicol antibiotics; clofibric acid, gemfibrozil, benzafibrate, pravastatin; oxacillin and cloxacillin) and positive mode for all others. Ions were acquired in multiple reaction monitoring (MRM) mode with a dwell time of 10 (50) ms. The mass spectrometer conditions were as follows: ion spray voltage: $5.5(-4.5) \mathrm{kV}$, curtain gas: $10 \mathrm{~L} / \mathrm{h}$, nebulizer gas: $60(50) \mathrm{L} / \mathrm{h}$, turbo gas: $50(60) \mathrm{L} / \mathrm{h}$, heated capillary temperature: $500{ }^{\circ} \mathrm{C}$, interface heater: $\mathrm{ON}$, and collisionally activated dissociation: 5 . ${ }^{*}$ note: when different from positive mode, values for negative modes are reported in parentheses.

After selecting the precursor ions, product ions were obtained and optimized with these four key parameters: declustering potential, entrance potential, collision energy, and collision cell exit potential by direct infusion of the pure analytes to the MS-MS compartment.

\subsubsection{Detection, quantification, and quality control}

Identification of pharmaceuticals was performed with HPLCMS/MS with multiple reaction monitoring (MRM), using the two highest characteristic precursor ion/product ion transition pairs. Compounds were identified using the LC retention time $\pm 30 \%$ of retention time of a standard. The MRM pair used for quantification is listed in Table 1.

Recovery experiments were performed on both DI and river water spiked with the $50 \mathrm{ng} / \mathrm{L}$ target analytes to estimate this method's percent recovery, which was determined by comparing the concentrations of the spiked DI and river water before and after SPE extraction. ${ }^{13} \mathrm{C}_{6}$-sulfamethazine was used as an internal standard for the quantification. The standard calibration curve was constructed by spiking waters with pharmaceutical standard solutions in the $0.5-2000 \mu \mathrm{g} / \mathrm{L}$ range, and the linearity of calibration curves was estimated by fitting a linear mode, least-squares regression analysis $(y=a+b x)$. The method detection limits (MDLs) were determined with the minimum concentration of analyte in the linear range with a signal-to noise ratio of $\geqslant 3: 1$ in a river water matrix. 
Table 1

Occurrence, usage, and toxicity data for 97 pharmaceuticals in six potential contamination sources

\begin{tabular}{|c|c|c|c|c|c|c|c|c|c|c|c|c|c|c|}
\hline & \multirow[t]{2}{*}{ MRM } & \multirow{2}{*}{$\begin{array}{l}\operatorname{Rec} \\
(\%)\end{array}$} & \multirow{2}{*}{$\begin{array}{l}\text { MDL } \\
\text { (ng/L) }\end{array}$} & \multirow{2}{*}{$\begin{array}{l}\text { Frequency } \\
(\%)\end{array}$} & \multirow{2}{*}{$\begin{array}{l}\operatorname{Max} \\
(\mathrm{ng} / \mathrm{L})\end{array}$} & \multicolumn{6}{|l|}{ Med } & \multirow{2}{*}{$\begin{array}{l}\text { Lowest PNEC } \\
\text { reported } \\
\text { (ng/L) }\end{array}$} & \multicolumn{2}{|c|}{ NHI 2005 medicine usage } \\
\hline & & & & & & $\begin{array}{l}\text { Drug production } \\
\text { facilities }(n=3) \\
(\mathrm{ng} / \mathrm{L})\end{array}$ & $\begin{array}{l}\text { Hospitals } \\
(n=6) \\
(\mathrm{ng} / \mathrm{L})\end{array}$ & $\begin{array}{l}\text { STPs } \\
(n=4) \\
(\mathrm{ng} / \mathrm{L}) \\
\end{array}$ & $\begin{array}{l}\text { Regional } \\
\text { discharges }(n=5) \\
(\mathrm{ng} / \mathrm{L})\end{array}$ & $\begin{array}{l}\text { Animal } \\
\text { husbandries }(n=2) \\
(\mathrm{ng} / \mathrm{L})\end{array}$ & $\begin{array}{l}\text { Aquacultures } \\
(n=3) \\
(\mathrm{ng} / \mathrm{L})\end{array}$ & & $\begin{array}{l}\text { Dose }^{\mathrm{e}} \\
\left(\times 10^{3}\right)\end{array}$ & Content $^{f}$ \\
\hline \multicolumn{15}{|l|}{ Sulfonamide antibiotics } \\
\hline Sulfanilamide & $\begin{array}{l}173.0 / \\
108.0\end{array}$ & 67.9 & 10 & 78 & 207 & 50 & 19 & 26 & 25 & 48 & ND & & 140 & $5 \mathrm{gm}$ \\
\hline Sulfaguanidine & $\begin{array}{l}215.0 / \\
156.0\end{array}$ & 57.4 & 1 & 43 & 145 & 4 & ND & 2 & ND & 26 & ND & & & \\
\hline Sulfadiazine & $\begin{array}{l}251.0 / \\
156.0\end{array}$ & 79.4 & 1 & 78 & 353 & 19 & 50 & 6 & 10 & 21 & ND & & 4,369 & $10 \mathrm{mg} / \mathrm{gm}$ \\
\hline Sulfamethoxazole ${ }^{\mathrm{b}}$ & $\begin{array}{l}254.0 / \\
156.0\end{array}$ & 69.1 & 1 & 96 & 5823 & 22 & 647 & 226 & 282 & 10 & 229 & 20000 & 16023 & $\begin{array}{l}80,400 \mathrm{mg} ; 20 \\
40 \mathrm{mg} / \mathrm{mL}\end{array}$ \\
\hline Sulfathiazole & $\begin{array}{l}256.0 / \\
156.0\end{array}$ & 79.2 & 1 & 48 & 9637 & ND & ND & 2 & ND & 4844 & 7 & & & \\
\hline Sulfamerazine & $\begin{array}{l}265.0 / \\
172.0\end{array}$ & 80 & 1 & 22 & 1660 & ND & 1 & ND & ND & 831 & ND & 116000 & & \\
\hline Sulfisoxazole & $\begin{array}{l}268.0 / \\
156.0\end{array}$ & 83 & 1 & 0 & ND & ND & ND & ND & ND & ND & ND & & & \\
\hline Sulfamethiazole & $\begin{array}{l}271.0 / \\
156.0\end{array}$ & 72.7 & 1 & 0 & ND & ND & ND & ND & ND & ND & ND & & & \\
\hline Sulfisomidine & $\begin{array}{l}279.0 / \\
186.0\end{array}$ & 80.8 & 1 & 13 & 2 & ND & ND & ND & ND & ND & ND & & & \\
\hline Sulfamethazine & $\begin{array}{l}279.0 / \\
186.1\end{array}$ & 81 & 1 & 35 & 370 & 1 & ND & 2 & ND & 77 & ND & $1.2 \times 10^{6^{d}}$ & & \\
\hline Sulfamethoxypyridazine & $\begin{array}{l}281.0 / \\
156.0\end{array}$ & 76.7 & 1 & 13 & 2 & ND & ND & ND & ND & ND & ND & & & \\
\hline Sulfamonomethoxine ${ }^{\mathrm{b}}$ & $\begin{array}{l}281.0 / \\
156.1\end{array}$ & 82.9 & 1 & 22 & 20 & ND & ND & ND & ND & ND & 2 & & & \\
\hline Sulfaquinoxaline & $\begin{array}{l}301.0 / \\
156.0\end{array}$ & 60.2 & 1 & 4 & 1.1 & ND & ND & ND & ND & ND & ND & & & \\
\hline Sulfadimethoxine & $\begin{array}{l}311.0 / \\
156.0\end{array}$ & 78.7 & 2.5 & 35 & 354 & ND & ND & 2 & ND & 17 & 21 & $248000^{\mathrm{d}}$ & 21 & $250,500 \mathrm{mg}$ \\
\hline Sulfinpyrazone & $\begin{array}{l}405.0 / \\
279.0\end{array}$ & 46 & 10 & 0 & ND & ND & ND & ND & ND & ND & ND & $10^{6}$ & 2619 & $100 \mathrm{mg}$ \\
\hline \multicolumn{15}{|l|}{ Tetracycline antibiotics } \\
\hline Tetracycline $\mathrm{a}^{\mathrm{a}, \mathrm{b}}$ & $\begin{array}{l}445.0 / \\
410.0\end{array}$ & 72.4 & 5 & 87 & 1570 & 5 & 59 & 21 & 38 & 1129 & ND & 90 & 7284 & $250 \mathrm{mg}, 125 \mathrm{mg} / \mathrm{gm}$ \\
\hline Oxytetracycline $\mathrm{e}^{\mathrm{a}, \mathrm{b}}$ & $\begin{array}{l}461.0 / \\
426.0\end{array}$ & 83 & 5 & 39 & 15133 & ND & ND & ND & ND & 8318 & 12 & 200 & 95 & $50,250 \mathrm{mg} ; 50 \mathrm{mg} / \mathrm{mL}$ \\
\hline Chlortetracycline $e^{\mathrm{a}, \mathrm{b}}$ & $\begin{array}{l}479.0 / \\
444.0\end{array}$ & 73.3 & 5 & 22 & 5637 & ND & ND & ND & ND & 2821 & 11 & $219000^{d}$ & 30 & $250 \mathrm{mg} ; 1,10 \mathrm{mg} / \mathrm{gm}$ \\
\hline \multicolumn{15}{|l|}{ Lincosamide antibiotics } \\
\hline Lincomycin $^{\mathrm{a}}$ & $\begin{array}{l}407.0 / \\
359.0\end{array}$ & 70.7 & 5 & 70 & 111667 & ND & 24 & 13 & 25 & 56760 & 70 & $>10^{6^{\mathrm{d}}}$ & 180 & $300 \mathrm{mg} / \mathrm{mL}$ \\
\hline Clindamycin & $\begin{array}{l}425.0 / \\
377.0\end{array}$ & 81.6 & 2.5 & 70 & 1150 & 10 & 341 & 51 & 199 & ND & ND & 500 & 34516 & $150 \mathrm{mg}, 150 \mathrm{mg} / \mathrm{mL}$ \\
\hline $\begin{array}{l}\text { Macrolide antibiotics } \\
\text { Spiramycin }\end{array}$ & $\begin{array}{l}422.5 / \\
145.0\end{array}$ & 36.7 & 10 & 0 & ND & ND & ND & ND & ND & ND & ND & & & \\
\hline Azithromycin & $\begin{array}{l}375.5 / \\
591.5\end{array}$ & 34.4 & 5 & 48 & 1067 & ND & 227 & 15 & ND & ND & ND & 150 & 296 & $250 \mathrm{mg}$ \\
\hline Oleandomycin ${ }^{a}$ & $\begin{array}{l}398.2 / \\
158.0\end{array}$ & 31.9 & 10 & 0 & ND & ND & ND & ND & ND & ND & ND & & & (continued on next page) \\
\hline
\end{tabular}




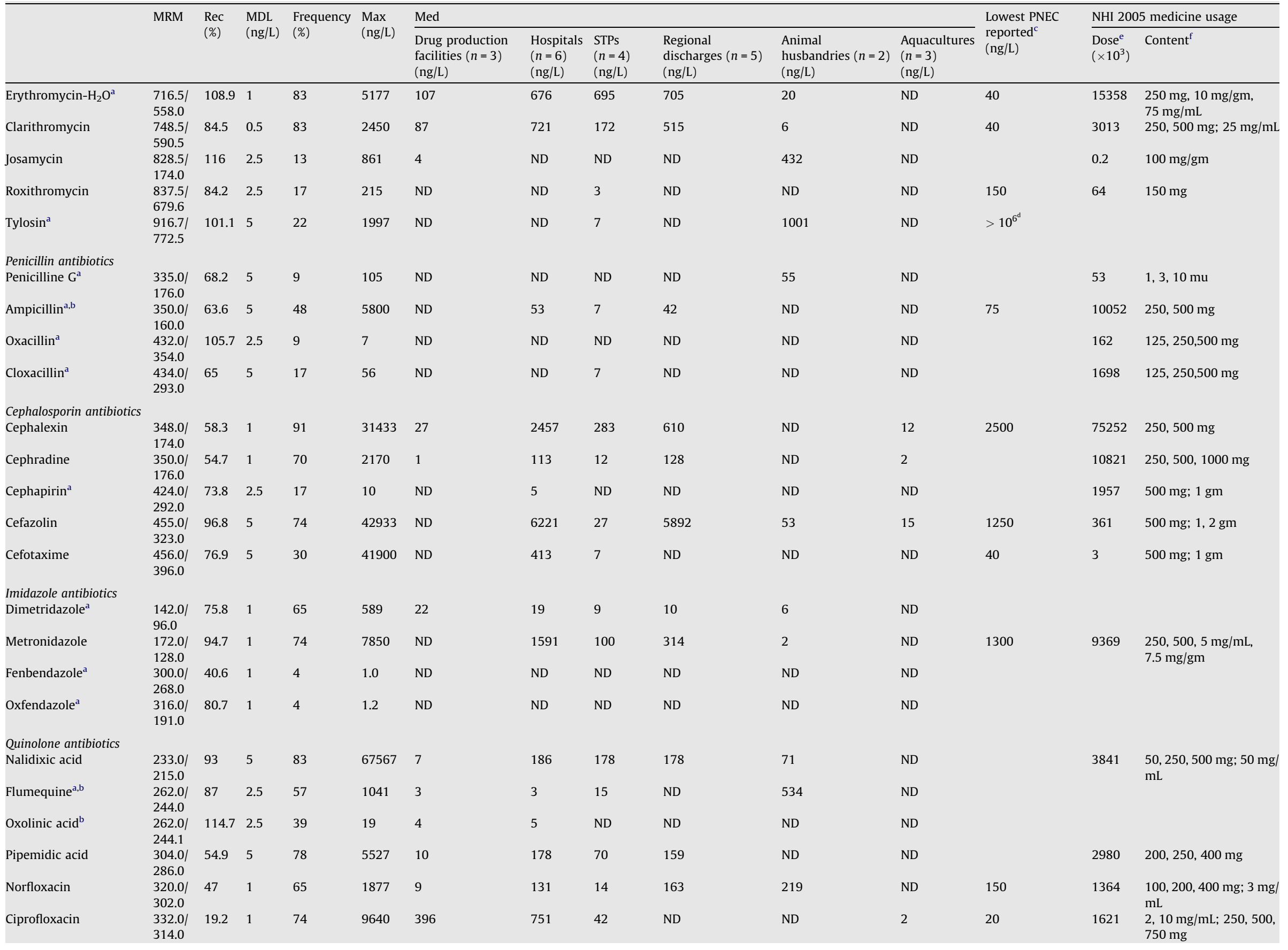




\begin{tabular}{|c|c|c|c|c|c|c|c|c|c|c|c|c|c|c|}
\hline Pefloxacin & $\begin{array}{l}334.0 / \\
316.0\end{array}$ & 75.3 & 5 & 30 & 950 & 12 & 62 & ND & ND & ND & ND & & 0.1 & $80 \mathrm{mg} / \mathrm{mL}$ \\
\hline Enrofloxacin & $\begin{array}{l}360.0 / \\
342.0\end{array}$ & 52.7 & 5 & 48 & 342 & 8 & 40 & ND & ND & 173 & ND & & & \\
\hline Ofloxacin & $\begin{array}{l}362.0 / \\
318.0\end{array}$ & 58.7 & 1 & 91 & 13633 & 853 & 1088 & 123 & 1065 & ND & 4 & 40 & 7025 & $\begin{array}{l}100,200,400 \mathrm{mg} ; 3 \mathrm{mg} / \\
\mathrm{mL}\end{array}$ \\
\hline Marbofloxacin & $\begin{array}{l}363.0 / \\
320.0\end{array}$ & 49.8 & 2.5 & 30 & 7 & 3 & 3 & ND & ND & ND & ND & & & \\
\hline Sarafloxacin ${ }^{\mathrm{a}}$ & $\begin{array}{l}386.0 / \\
368.0\end{array}$ & 54.6 & 1 & 17 & 17 & 4 & ND & ND & ND & ND & ND & & & \\
\hline Difloxacin & $\begin{array}{l}400.0 / \\
382.0\end{array}$ & 64.6 & 5 & 9 & 10 & 6 & ND & ND & ND & ND & ND & & & \\
\hline \multicolumn{15}{|c|}{ Chloramphenicol antibiotics } \\
\hline Chloramphenicol & $\begin{array}{l}323.0 / \\
152.0\end{array}$ & 120 & 0.5 & 52 & 114 & ND & 1 & 50 & 1 & ND & ND & 1600 & 6156 & $\begin{array}{l}10,250 \mathrm{mg} / \mathrm{gm} ; 2.5,5 \\
125,250 \mathrm{mg} / \mathrm{mL}\end{array}$ \\
\hline Thiamphenicol ${ }^{\mathrm{a}, \mathrm{b}}$ & $\begin{array}{l}354.0 / \\
290.0\end{array}$ & 94.2 & 0.5 & 70 & 192 & 1 & 4 & 138 & 7 & ND & ND & & 5048 & $\begin{array}{l}250,500 \mathrm{mg} ; 100 \mathrm{mg} / \\
\mathrm{gm}\end{array}$ \\
\hline Florfenicol $^{\mathrm{a}, \mathrm{b}}$ & $\begin{array}{l}358.0 / \\
338.0\end{array}$ & 108.7 & 0.5 & 9 & 36 & ND & ND & ND & ND & ND & 8 & & & \\
\hline \multicolumn{15}{|l|}{ Other antibiotics } \\
\hline Morantel $^{\mathrm{a}}$ & $\begin{array}{l}221.0 / \\
123.0\end{array}$ & 115.3 & 2.5 & 0 & ND & ND & ND & ND & ND & ND & ND & & & \\
\hline Carbadox ${ }^{\mathrm{a}}$ & $\begin{array}{l}263.0 / \\
231.0\end{array}$ & 92.5 & 5 & 4 & 6 & ND & ND & ND & ND & ND & ND & & & \\
\hline Trimethoprim ${ }^{\mathrm{a}}$ & $\begin{array}{l}291.0 / \\
261.0\end{array}$ & 82.7 & 2.5 & 87 & 2993 & ND & 1040 & 321 & 803 & 32 & 85 & 1000 & 13042 & $80,400 \mathrm{mg}$ \\
\hline Colchicines & $\begin{array}{l}400.0 / \\
358.0\end{array}$ & 134.3 & 5 & 17 & 37 & ND & 9 & ND & ND & ND & ND & & & \\
\hline \multicolumn{15}{|l|}{$\beta$-agonists } \\
\hline Terbutaline & $\begin{array}{l}226.0 / \\
152.0\end{array}$ & 15.5 & 5 & 35 & 53 & ND & 38 & 9 & ND & ND & ND & 240000 & 16564 & $\begin{array}{l}2.5,5 \mathrm{mg} ; 0.2,2.5 \mathrm{mg} / \\
\mathrm{mL}\end{array}$ \\
\hline Tulobuterol & $\begin{array}{l}230.0 / \\
156.0\end{array}$ & 86.8 & 1 & 13 & 137 & 1 & ND & ND & ND & ND & ND & & & \\
\hline Salbutamol & $\begin{array}{l}240.0 / \\
222.0\end{array}$ & 11.7 & 0.5 & 61 & 173 & 1 & 22 & 5 & 9 & ND & ND & 240000 & 22894 & $\begin{array}{l}2,4 \mathrm{mg} ; 0.2,0.4,0.5 \\
1 \mathrm{mg} / \mathrm{mL}\end{array}$ \\
\hline Clenbuterol $^{a}$ & $\begin{array}{l}277.0 / \\
168.0\end{array}$ & 85 & 2.5 & 0 & ND & ND & ND & ND & ND & ND & ND & & & \\
\hline Brombuterol & $\begin{array}{l}367.0 / \\
293.0\end{array}$ & 76.7 & 2.5 & 0 & ND & ND & ND & ND & ND & ND & ND & & & \\
\hline Ractopamine & $\begin{array}{l}302.0 / \\
284.0\end{array}$ & 78.6 & 5 & 4 & 48 & ND & ND & ND & ND & ND & ND & & & \\
\hline \multicolumn{15}{|l|}{ Estrogens } \\
\hline P-Estradiol ${ }^{\mathrm{a}}$ & $\begin{array}{l}255.0 / \\
159.0\end{array}$ & 65.8 & 10 & 0 & ND & ND & ND & ND & ND & ND & ND & 0.020 & 19106 & $0.2,3 \mathrm{mg} ; 2,5$ \\
\hline Estriol $^{\mathrm{a}}$ & $\begin{array}{l}271.0 / \\
253.0\end{array}$ & 96 & 5 & 39 & 22633 & ND & 4651 & ND & 4648 & 7169 & ND & 0.8 & 149 & $0.1,0.5,10 \mathrm{mg} ; 1 \mathrm{mg} / \mathrm{gm}$ \\
\hline Estrone & $\begin{array}{l}271.0 / \\
253.1\end{array}$ & 107.3 & 10 & 4 & 345 & ND & ND & ND & ND & ND & ND & $18^{\mathrm{d}}$ & 15 & $1.25 \mathrm{mg}$ \\
\hline 17a-ethynylestradiol & $\begin{array}{l}279.0 / \\
159.0\end{array}$ & 54.5 & 10 & 4 & 487 & ND & ND & ND & ND & ND & ND & 0.02 & 801 & $0.035 \mathrm{mg}$ \\
\hline Progesterone $^{\mathrm{a}}$ & $\begin{array}{l}315.0 / \\
109.0\end{array}$ & 59.9 & 5 & 22 & 53 & ND & 9 & ND & ND & ND & ND & & 2160 & $\begin{array}{l}25,50,125 \mathrm{mg} / \mathrm{mL} ; 10 \\
20,50 \mathrm{mg}\end{array}$ \\
\hline$\beta$-blockers & & & & & & & & & & & & & & \\
\hline Propranolol & $\begin{array}{l}260.0 / \\
183.0\end{array}$ & 69 & 2.5 & 43 & 171 & ND & 42 & 50 & ND & ND & ND & 500 & 156637 & $10,40 \mathrm{mg}$ \\
\hline Atenolol & $\begin{array}{l}267.0 / \\
190.0\end{array}$ & 35.2 & 5 & 78 & 2260 & 16 & 1607 & 411 & 1025 & 52 & ND & $10 \times 10^{6}$ & & (continued on next nage) \\
\hline
\end{tabular}




\begin{tabular}{|c|c|c|c|c|c|c|c|c|c|c|c|c|c|c|}
\hline & \multirow[t]{2}{*}{ MRM } & \multirow{2}{*}{$\begin{array}{l}\operatorname{Rec} \\
(\%)\end{array}$} & \multirow{2}{*}{$\begin{array}{l}\mathrm{MDL} \\
(\mathrm{ng} / \mathrm{L})\end{array}$} & \multirow{2}{*}{$\begin{array}{l}\text { Frequency } \\
\text { (\%) }\end{array}$} & \multirow{2}{*}{$\begin{array}{l}\operatorname{Max} \\
(\mathrm{ng} / \mathrm{L})\end{array}$} & \multicolumn{6}{|l|}{ Med } & \multirow{2}{*}{$\begin{array}{l}\text { Lowest PNEC } \\
\text { reported }^{\mathrm{C}}(\mathrm{ng} / \mathrm{L})\end{array}$} & \multicolumn{2}{|c|}{ NHI 2005 medicine usage } \\
\hline & & & & & & $\begin{array}{l}\text { Drug production } \\
\text { facilities }(n=3) \\
(\mathrm{ng} / \mathrm{L})\end{array}$ & $\begin{array}{l}\text { Hospitals } \\
(n=6) \\
(\mathrm{ng} / \mathrm{L})\end{array}$ & $\begin{array}{l}\text { STPs } \\
(n=4) \\
(\mathrm{ng} / \mathrm{L})\end{array}$ & $\begin{array}{l}\text { Regional } \\
\text { discharges }(n=5) \\
(\mathrm{ng} / \mathrm{L})\end{array}$ & $\begin{array}{l}\text { Animal } \\
\text { husbandries }(n=2) \\
\text { (ng/L) }\end{array}$ & $\begin{array}{l}\text { Aquacultures } \\
(n=3) \\
(\mathrm{ng} / \mathrm{L})\end{array}$ & & $\begin{array}{l}\text { Dose } e^{e} \\
\left(\times 10^{3}\right)\end{array}$ & Content $^{\mathrm{f}}$ \\
\hline Metoprolol & $\begin{array}{l}268.0 / \\
191.0\end{array}$ & 70 & 5 & 52 & 631 & ND & 145 & 161 & ND & ND & ND & 8800 & 1561 & $50,100 \mathrm{mg}$ \\
\hline Sotalol & $\begin{array}{l}273.0 / \\
255.0\end{array}$ & 68.9 & 10 & 17 & 619 & ND & ND & 11 & ND & ND & ND & & 221 & $80,160 \mathrm{mg}$ \\
\hline Acebutolol & $\begin{array}{l}337.0 / \\
319.0\end{array}$ & 105.1 & 2.5 & 70 & 1323 & 6 & 185 & 223 & 223 & ND & ND & & 8017 & $100,200,400 \mathrm{mg}$ \\
\hline \multicolumn{15}{|l|}{ Psychostimulants } \\
\hline Caffeine & $\begin{array}{l}195.0 / \\
138.0\end{array}$ & 115.3 & 0.5 & 96 & 23967 & 5 & 15600 & 243 & 7560 & 45 & 36 & $>10 \times 10^{6}$ & 396 & $10,20,25,50,100 \mathrm{mg}$ \\
\hline \multicolumn{15}{|l|}{ Vasodilators } \\
\hline Pentoxifylline & $\begin{array}{l}279.0 / \\
181.0\end{array}$ & 84.6 & 1 & 70 & 9767 & 1370 & 302 & 90 & 188 & ND & ND & & 33613 & $100,400 \mathrm{mg} ; 20 \mathrm{mg} / \mathrm{mL}$ \\
\hline \multicolumn{15}{|l|}{ Psychiatric drugs } \\
\hline Carbamazepine & $\begin{array}{l}237.0 / \\
194.0\end{array}$ & 93.4 & 2.5 & 87 & 10933 & 7810 & 163 & 152 & 138 & 3 & ND & 25000 & 30019 & $\begin{array}{l}100,200,300,400 \mathrm{mg} \\
20 \mathrm{mg} / \mathrm{mL}\end{array}$ \\
\hline Fluoxetin & $\begin{array}{l}310.0 / \\
148.0\end{array}$ & 37.1 & 5 & 17 & 371 & 154 & ND & ND & ND & 13 & ND & & 14221 & $10,20 \mathrm{mg}$ \\
\hline Paroxetine & $\begin{array}{l}330.0 / \\
192.0\end{array}$ & 31.5 & 2.5 & 22 & 35 & 3 & ND & ND & ND & ND & ND & & 6571 & $12.5,20,30 \mathrm{mg}$ \\
\hline \multicolumn{15}{|l|}{ NSAIDs } \\
\hline Acetaminophen & $\begin{array}{l}152.0 / \\
110.0\end{array}$ & 84.9 & 5 & 96 & 100433 & 9 & 36950 & 16 & 8060 & 12 & 21 & 9200 & 568839 & $500 \mathrm{mg} ; 24 \mathrm{mg} / \mathrm{mL}$ \\
\hline Mepirizole & $\begin{array}{l}235.0 / \\
220.0\end{array}$ & 104.1 & 5 & 0 & ND & ND & ND & ND & ND & ND & ND & & 2402 & \\
\hline Famotidine & $\begin{array}{l}338.0 / \\
259.0\end{array}$ & 26.9 & 5 & 61 & 252 & 25 & 94 & 8 & 14 & ND & ND & & & \\
\hline Ibuprofen & $\begin{array}{l}205.0 / \\
161.0\end{array}$ & 62.3 & 5 & 96 & 14500 & 101 & 282 & 1758 & 747 & 836 & 50 & 5000 & 131359 & $200,400,600 \mathrm{mg}$ \\
\hline Naproxen & $\begin{array}{l}229.0 / \\
185.0\end{array}$ & 61.9 & 2.5 & 70 & 8463 & ND & 470 & 548 & 278 & 1766 & ND & 37000 & 22655 & $100,250 \mathrm{mg}$ \\
\hline Fenoprofen & $\begin{array}{l}241.0 / \\
197.0\end{array}$ & 77 & 7.5 & 43 & 214 & ND & ND & 24 & 0 & 8 & ND & & 1 & $200 \mathrm{mg}$ \\
\hline Ketoprofen & $\begin{array}{l}253.0 / \\
209.0\end{array}$ & 78.3 & 10 & 35 & 319 & ND & ND & ND & 0 & 164 & ND & $15.6 \times 10^{6^{d}}$ & & \\
\hline Fenbufen & $\begin{array}{l}253.0 / \\
209.1\end{array}$ & 67.3 & 10 & 22 & 43 & 31 & 15 & ND & ND & ND & ND & & 2923 & $200,300 \mathrm{mg}$ \\
\hline Fenoprop & $\begin{array}{l}267.0 / \\
195.0\end{array}$ & 108.5 & 2.5 & 0 & ND & ND & ND & ND & ND & ND & ND & & & \\
\hline Diclofenac & $\begin{array}{l}296.0 / \\
252.0\end{array}$ & 67.4 & 2.5 & 91 & 29767 & 20733 & 286 & 61 & 184 & 4 & 4 & 10000 & 214672 & $\begin{array}{l}25,50,75,100 \mathrm{mg} \\
25 \mathrm{mg} / \mathrm{mL} ; 10 \mathrm{mg} / \mathrm{gm}\end{array}$ \\
\hline Piroxicam & $\begin{array}{l}330.0 / \\
266.0\end{array}$ & 76.9 & 1 & 0 & ND & ND & ND & ND & ND & ND & ND & & 14920 & $\begin{array}{l}10,20 \mathrm{mg} ; 20 \mathrm{mg} / \mathrm{mL} \\
5,10 \mathrm{mg} / \mathrm{gm}\end{array}$ \\
\hline Indomethacin & $\begin{array}{l}356.0 / \\
312.0\end{array}$ & 68.3 & 5 & 22 & 231 & ND & ND & ND & ND & ND & ND & & 14218 & $\begin{array}{l}25,50,75,100 \mathrm{mg} \\
10 \mathrm{mg} / \mathrm{gm}\end{array}$ \\
\hline \multicolumn{15}{|c|}{ Lipid-regulator/cholesterol-lowering drug } \\
\hline Clofibic acid & $\begin{array}{l}213.0 / \\
127.0\end{array}$ & 93.3 & 1 & 65 & 2400 & ND & 9 & 154 & 35 & ND & ND & 12000 & & \\
\hline Gemfibrozil & $\begin{array}{l}249.0 / \\
127.0\end{array}$ & 74.6 & 1 & 52 & 10393 & 443 & 134 & ND & 36 & ND & ND & 100000 & 25015 & $300,600 \mathrm{mg}$ \\
\hline
\end{tabular}




\subsection{Taiwan's medication usage data}

Data concerning Taiwan's medication usage in 2005 (the most recent data available) were recorded by the National Health Research Institutes (NHRI) and underwent statistical analysis (Table 1). Data from years prior to 2005 were also checked, and very similar trends were observed. In Taiwan, approximately 1578 distinct medicines are used annually in the National Health Insurance (NHI) program, which provides comprehensive health care for all Taiwanese citizens. The NHI was implemented in March 1995, and by the end of 2005 , more than $97.5 \%$ of the population had enrolled in the program, while $91 \%$ of health care providers had contracted with the Bureau of NHI (BNHI) (Department of Health, 2008). The NHRI maintains a comprehensive, current database on quantities and types of drugs used in hospital and clinic participants in the NHI program, thus providing an excellent representation of medication use patterns for the entire Taiwanese population.

\section{Results and discussion}

The 97 target compounds are listed in Table 1 with their generic classes, MRM pair (for quantification), percent recoveries, and method detection limits (MDLs) in environmental aqueous samples. The majority (86\%) of the target analytes were $>50 \%$ recovered with this novel method, while more than half had recoveries ranging from $75-120 \%$. MDLs ranged from 0.5 to $10 \mathrm{ng} / \mathrm{L}$ with linearity $>0.9911$. Recoveries and MDLs for DI water were also performed (data not shown), with similar results but slightly better recoveries and lower MDLs for DI water in general.

Table 1 summarizes detection frequencies and median values for all compounds found in each potential pharmaceutical contamination source, as well as each compound's maximum concentration out of all 23 sites studied. A large number of pharmaceuticals (84 out of 97) were detected at least once during this study; only six antibiotics, two $\beta$-agonists, one estrogen, three NSAIDs, and one lipid-regulator/cholesterol-lowering drug were not seen at all. For detected compounds, measured maximum concentrations ranged from a few $\mathrm{ng} / \mathrm{L}$ to approximately $0.1 \mathrm{mg} / \mathrm{L}$. Compounds that were frequently detected also had relatively higher maximum concentrations. The two exceptions were estriol and gemfibrozil, which were detected only half of the time but had concentrations up to 22633 and $10393 \mathrm{ng} / \mathrm{L}$ (seen in hospital effluents). This likely represents the usage of these two specific medicines in their source hospitals.

The 97 target compounds comprised a total of 18 distinct pharmaceutical classes (ten classes of antibiotics and eight other classes of pharmaceuticals). For each class, Fig. 1a shows frequency of detection and percent of total measured concentration (normalized to number of sampling locations in each of the six potential sources). It should be noted that the total mass contribution of pharmaceuticals from each source/compound group was not quantified because volumetric flows were not measured during sampling. Eighteen classes were detected in samples from at least ten of 23 sampling sites. Estrogens (43\%) were the least frequently detected class, followed by penicillin antibiotics (57\%). Sulfonamides, quinolones, and NSAIDs were the three most ubiquitous classes and were present in all samples analyzed. NSAIDs (26.7\%), lincosamides (18.5\%), cephalosporins (13.8\%), and quinolones (12.4\%) dominated the other drug classes in concentration, together comprising $>70 \%$ of the total normalized concentrations measured (Fig. 1a). Penicillins, chloramphenicol antibiotics, and $\beta$-agonists contributed the least mass, together comprising $<0.5 \%$ of the total normalized measured concentrations. 
Fig. 1b depicts each class's distribution among six potential contamination sources. Lincosamides were almost entirely (98\%) derived from animal husbandry effluents. The majority of the cephalosporins and quinolones were from hospitals (73\% and $12 \%$, respectively) and drug production facilities (24\% and $83 \%$ ). Concentrations of NSAIDs were more evenly distributed among effluents of hospitals (53\%), drug production facilities (21\%), and regional discharges (16.5\%). NSAIDs are widely used for humans, and many are available without a prescription, which may explain their prevalence in regional discharges. According to the National Health Insurance Research database (NHIR) (National Research Health Research Database, 2008), many NSAIDs are among the top-used medicines in hospitals belonging to the NHI (i.e. acetaminophen, ibuprofen, diclofenac, and naproxen), with frequencies of use reflecting the detection frequencies reported here.

Penicillin-family medications were detected at trace amounts only. However a Taiwanese government investigation of antibiot- ics usage in 2006 (National Antibiotics, 2006), reported that in 2004-2005, penicillins and cephalosporins were manufactured or imported in greater amounts than all other human-use antibiotics, and there is no evidence to believe that medication usage patterns have dramatically shifted away from these since that time. Cephalosporins are also used in veterinary medicine, which should further increase their concentrations in hospital effluents. However the apparent contradiction between the significant quantities of penicillins used, manufactured, or imported, and the trace or entirely absent amounts found in the aqueous environment can be explained by their easily hydrolyzed nature, as reported by Hirsch et al. (1999).

Fig. 2 lists the pharmaceuticals found at each source and describes the distribution of the various drug classes for each source. The ten antibiotic classes were grouped into one, while classes with fewer members were combined into an "other pharmaceuticals" category, which included psychiatric drugs, vasodilators,
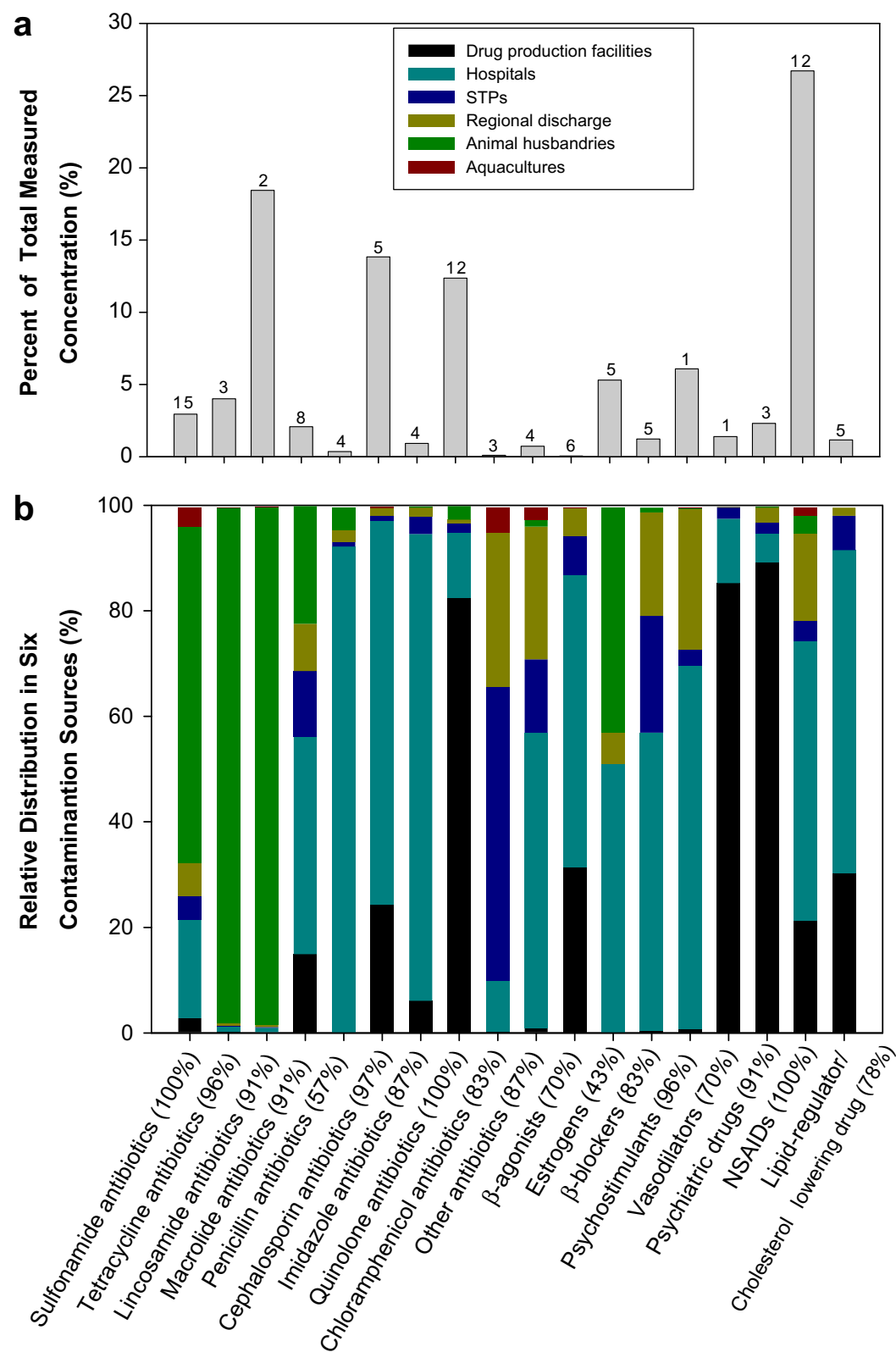

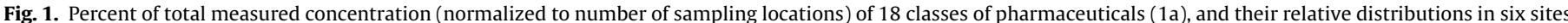

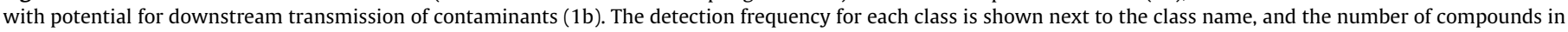
each class is noted above the bar. 


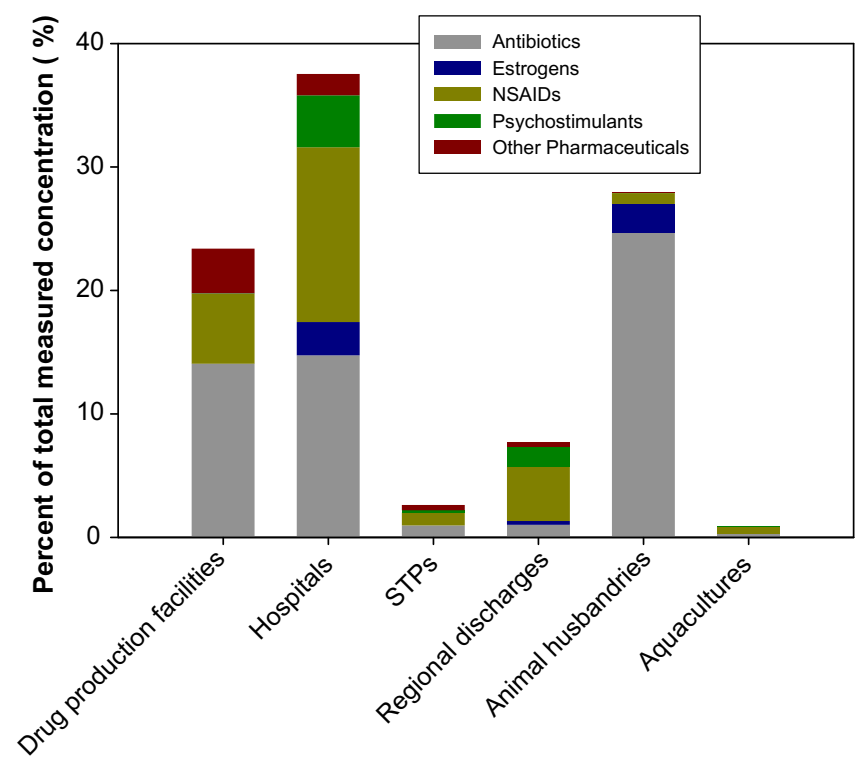

Fig. 2. The proportion of pharmaceuticals found at six potential contamination sources. The percent of total measured concentration (normalized to number of sampling locations) and the distribution of each pharmaceutical class are indicated for each source.

$\beta$-blockers, lipid-regulator/cholesterol-lowering drugs, and $\beta$-agonists. The highest total normalized drug concentrations were found in waste streams from hospitals (37.5\%), followed by animal husbandries (27.9\%) and drug production facilities (23.4\%). Aquaculture effluents were the most free of pharmaceutical contamination, contributing $<1.0 \%$ of the total normalized concentrations. In hospital effluents, antibiotics (39.3\%), NSAIDs (37.7\%), psychostim- ulants (11.2\%), and estrogens (7.2\%) were the four dominant groups. In animal husbandry effluents, antibiotics comprised $88.5 \%$ of the water composition, while estrogens accounted for an additional $8.2 \%$. In drug production facility effluents, significant amounts of vasodilators (5.0\%) and psychiatric drugs (8.8\%) were found, in addition to the ubiquitous antibiotics and NSAIDs. Compounds in the "other pharmaceuticals" group distributed similarly in waste effluents, with the exception of $\beta$-agonists, which contributed only $0.05 \%$ to the total concentration. The medications in the "other pharmaceuticals" group are used exclusively in humans, accounting for their occurrence in hospitals, STPs, and regional discharges, and their lower concentrations or complete absence from animal husbandry and aquaculture effluents.

Fig. 3 shows the 27 most frequently detected (>70\%) compounds. Multiple pharmaceutical classes were represented, of which $37.0 \%$ had medians $>100 \mathrm{ng} / \mathrm{L}$ and $77.8 \%$ had 75 th-percentile concentrations $>100 \mathrm{ng} / \mathrm{L}$. Except for three, all of the 27 compounds had maximum values $>1000 \mathrm{ng} / \mathrm{L}$, the exceptions being sulfanilamide, sulfadiazine, and thiamphenicol, which had the lowest maximum concentration (192 ng/L). The high concentrations observed indicate that significant amounts of pharmaceuticals were present in these six potential contamination sources.

Sulfamethoxazole, caffeine, acetaminophen, and ibuprofen were the most frequently detected compounds, present in all effluents but one, at concentrations up to 5823, 23957, 100433 and, $14500 \mathrm{ng} / \mathrm{L}$, respectively. Cephalexin, ofloxacin and diclofenac were the next most often detected compounds, with concentrations up to 31433,13633 , and $29767 \mathrm{ng} / \mathrm{L}$. The prevalence of these seven medications is consistent with findings of previous investigators. For instance, Kolpin et al. (2002) targeted 95 compounds in a survey of 139 streams in the United States and detected caffeine, acetaminophen and sulfamethoxazole at frequencies of $70.6 \%, 23.8 \%$ and $19.0 \%$. Managaki et al. (2007) reported that in Vietnamese urban drainage samples, sulfamethoxazole $(0.190-0.369 \mu \mathrm{g} / \mathrm{L})$ was

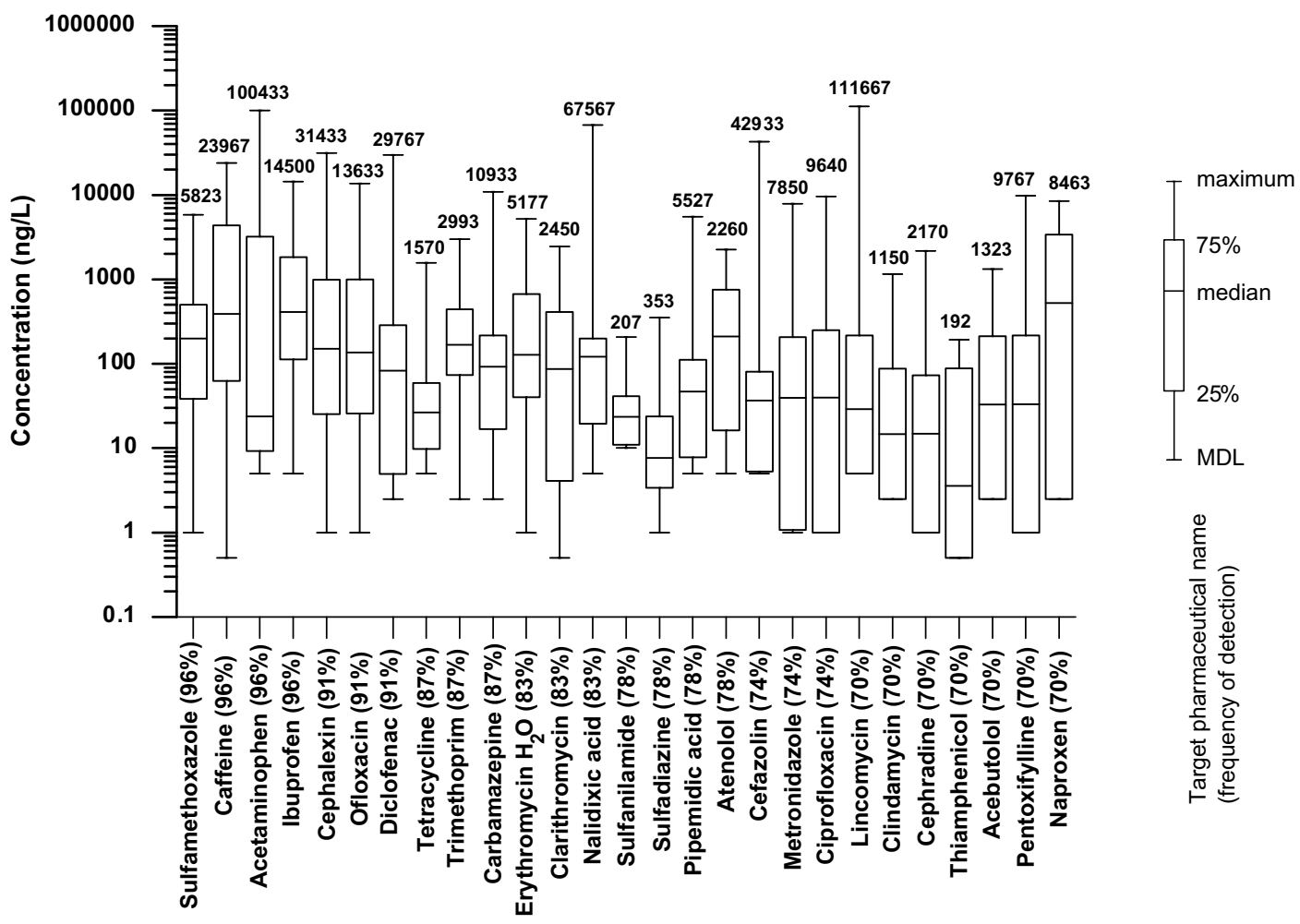

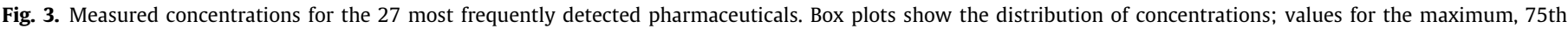

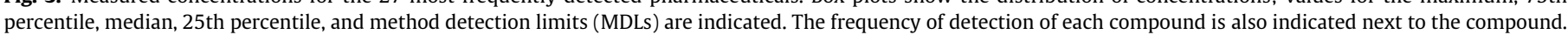


present at concentrations higher than all other pharmaceuticals assayed, indicating a primary derivation from human use. Moreover, Sacher et al. (2001) showed that of 60 pharmaceuticals targeted, sulfamethoxazole was one of the most frequently detected antibiotics, with concentrations up to $0.410 \mu \mathrm{g} / \mathrm{L}$ in groundswell in Baden-Württemberg, Germany. Brown et al. (2006) studied eleven antibiotics and frequently found sulfamethoxazole and ofloxacin in hospital effluents, at concentrations ranging from 400 to 2100 and 4900 to $35500 \mathrm{ng} / \mathrm{L}$. Another study (Gomez et al., 2006) of hospital effluents also revealed high concentrations of acetaminophen, ibuprofen, and diclofenac (up to 29, 151 and $1.9 \mu \mathrm{g} / \mathrm{L}$ ).

Variations in MDLs and recoveries can influence detection frequencies and detected concentrations. For example, ciprofloxacin (a quinolones) was only 19.2\% recovered with our method, with concentrations up to $9640 \mathrm{ng} / \mathrm{L}$. If an extraction method more suitable for ciprofloxacin were applied, we would expect to see increased detection frequency and aqueous concentrations. However, although results are not perfect for all compounds assayed, the novel pharmaceutical screen described here obviates the need to perform individually tailored analyses for each of the many target drugs.

Detection frequencies and maximum effluent concentrations in many cases correlate well with Taiwan's medication usage patterns, as recorded by the NHRI (Table 1). A more precise correlation between these contamination metrics and actual amounts of medication prescribed would have been even more illuminating, but this comparison presents several insurmountable hurdles. First, the NHRI data records only the number of times a medication was prescribed and does not provide doses. Second, although individual hospitals or pharmacies may have collected similar data for internal use, and although drug companies themselves certainly kept dosage data for marketing purposes, such data are likely to be proprietary, in multiple different formats, or otherwise unavailable for study. Therefore only the raw medication usage data are presented (Table 1). Despite the bias introduced by using prescription data as a surrogate for actual dosages, we demonstrated that in general, medicines that were often prescribed correlated with higher detection frequencies and observed concentrations in many human-derived waste effluents. Analysis of usage data from the year 2005 for 60 of our target compounds revealed that acetaminophen, an NSAID, was the most-used medicine of 2005, with a total of 568.8 million doses prescribed ( $500 \mathrm{mg}, 24 \mathrm{mg} / \mathrm{mL}$ ). Diclofenac, atenolol, ibuprofen, cephalexin, clindamycin, pentoxifylline, and carbamazepine were also frequently prescribed, with usages in the top 100 of 1578 distinct drugs. These eight medicines were among the most frequently detected groups (Fig. 3) in this study. All were found at relatively high concentrations in humanimpacted contamination sources (drug production facilities, hospitals, STPs and regional discharges), with maximum concentrations ranging from 1150 to $100433 \mathrm{ng} / \mathrm{L}$.

Taiwan has long regulated the use of pharmaceuticals in agriculture. Twenty-five antibiotics, one $\beta$-agonist, and two estrogens are listed in the government's Maximum Residues Limits for Veterinary Drugs (Department of Health, 2008) and Aquaculture Pharmaceutical Use Guidance (Council of Agriculture, 2008) regulations and are noted with superscripts 'a' and 'b' in Table 1. When regulated veterinary medicines were queried, only 12 antibiotics were detected in agricultural effluents: erythromycin$\mathrm{H}_{2} \mathrm{O}$, tylosin, florfenicol, flumequine, lincomycin, chlortetracycline, oxytetracycline, tetracycline, sulfadimethoxine, sulfamonomethoxine, dimetridazole, and trimethoprim. Each compound fell into one of the ten antibiotics classes studied, and all ten classes were represented; for veterinary medications, these 12 compounds were taken as representatives for each class. Other investigators have also reported the use and occurrence of these 12 drugs in agriculture (Brown et al., 2006; Managaki et al., 2007). In our samples, lincomycin and the tetracyclines were the most prevalent antibiotics in the two animal husbandry effluents, with concentrations up to 111,667 (lincomycin) and $15,133 \mathrm{ng} / \mathrm{L}$ (oxytetracycline). The remaining antibiotics occurred in much lower concentrations, especially in aquaculture waste streams. These findings are consistent with a previous study of similar antibiotic classes in eight dairy effluents in New Mexico, in which lincomycin was the only antibiotic detected (concentration 700-6600 ng/L) (Brown et al., 2006).

The actual number of distinct pharmaceuticals detected ranged from 36-42, 41-54, 39-49, 30-49, 14-30, and 13-28, for samples from drug production facilities, hospitals, STPs, regional discharges, animal husbandries, and aquacultures, respectively. These counts, in combination with the fact that the majority of the target compounds were human-use medicines, demonstrate that our targeted contaminants were derived mainly from human activities. In contrast, agricultural industries use smaller amounts of pharmaceuticals, and agriculture-specific medications were detected at correspondingly low levels or not at all.

The many types and high concentrations of pharmaceuticals identified in the waste streams sampled indicate that our target compounds were not fully removed in wastewater treatment processes. Further studies on treatment efficiencies and natural attenuation processes will be needed to determine the fate of these pharmaceuticals in receiving environmental waters.

\subsection{Risk assessment}

The risk associated with residual pharmaceuticals transmitted from effluents to receiving water bodies is usually characterized as the ratio of environmental concentrations (predicted or measured) to predicted no-effect concentrations (PNECs). A PNEC is normally estimated by dividing the lowest no-observed-effect concentration (NOEC) for the most sensitive species by a safety factor (Carlsson et al., 2006). However, if NOEC data is not available, values such as minimal inhibitory concentrations (MICs), lowest observable effect concentrations (LOEC), or toxicity thresholds (TT) can be used to estimate PNECs (Jones et al., 2002; Kummerer and Henninger, 2003; Quinn et al., 2008). PNEC data for target pharmaceuticals are compiled and compared in Table 1, which lists the lowest PNEC values reported in the literature; when PNEC values were not available, the lowest acute and chronic $\mathrm{LC}_{50}$ and $\mathrm{EC}_{50}$ data were listed as references.

PNEC values for a third of our target analytes are reported, and significant numbers of compounds had effluent concentrations in excess of or very close to PNEC. Three estrogens (estriol, estrone, and $17 \alpha$-ethynylestradiol), three NSAIDs (acetaminophen, ibuprofen, and diclofenac), and 16 antibiotics occurred at levels higher than the PNEC reported; estrogens (estriol in particular) exceeded the $\mathrm{EC}_{50}$ by more than a factor of $10^{4}$. Maximum concentrations of many $\beta$-blockers, psychiatric drugs, and lipid regulators were less than a factor of 10 below reported PNECs. The ubiquity and high environmental concentrations which we report here are causes for concern and further investigation, as many of these medications are frequently prescribed. In addition, effluents from drug production facilities, hospitals, STPs, regional discharges, animal husbandries and aquacultures are the six most important potential contamination sources of pharmaceuticals in Taiwanese surface water systems, implying a high likelihood of similarly ubiquitous occurrence and high concentrations of pharmaceuticals in downstream surface water systems. Countries with similar economic activities and ways of life are likely to display similar contamination profiles. Key pharmaceuticals with high ubiquity and concentration may be investigated for their potential application as early indicators of total pharmaceutical burden; acetaminophen, the most frequently used, identified, and concentrated contaminant 
here (median concentrations >PNEC), is one such compound. With many other pharmaceuticals exceeding PNEC values at the source of contamination (and many more without any aquatic toxicity data at all), further investigations should include studies of their occurrence in receiving waters and the possible ecological and human health effects which may result.

\section{Acknowledgments}

Financial support was generously provided by the Environmental Protection Administration, Executive Yuan, Taiwan, ROC, under Grant No. EPA-96-G106-02-237, and by the National Science Council, Taiwan, ROC, under Grant No. NSC96-2221-E-002-042-MY3.

\section{Appendix A. Supplementary material}

Supplementary material associated with this article can be found, in the online version, at doi:10.1016/j.chemosphere.2008. 08.027.

\section{References}

Brown, K.D. Kulis, J., Thomson, B., Chapman, T.H., Mawhinney, D.B., 2006. Occurrence of antibiotics in hospital, residential, and dairy, effluent, municipal wastewater, and the Rio Grande in New Mexico. Science of the Total Environment 366, 772-783.

Carlsson, C., Johansson, A.K., Alvan, G., Bergman, K., Kuhler, T., 2006. Are pharmaceuticals potent environmental pollutants? Part I: Environmental risk assessments of selected active pharmaceutical ingredients. Science of the Total Environment 364, 67-87.

Council of Agriculture, 2008. http://www.coa.gov.tw/show_lawcommond.php? serial=9_webuser1_20050512115944\&code=A11\&type=A\&print=1 (accessed April 2008).

Department of Health, 2008. http://dohlaw.doh.gov.tw/Chi/FLAW/FLAWDAT0202. asp (accessed April 2008).

Directorate-General of Budget, Accounting and Statistics, 2008. http:// www.dgbas.gov.tw/ct.asp?xItem=13213\&CtNode=3504 (accessed April 2008).

Golet, E.M., Alder, A.C., Giger, W., 2002. Environmental exposure and risk assessment of fluoroquinolone antibacterial agents in wastewater and river water of the Glatt Valley Watershed, Switzerland. Environmental Science \& Technology 36, 3645-3651.

Gomez, M.J., Petrovic, M., Fernandez-Alba, A.R., Barcelo, D., 2006. Determination of pharmaceuticals of various therapeutic classes by solid-phase extraction and liquid chromatography-tandem mass spectrometry analysis in hospital effluent wastewaters. Journal of Chromatography A 1114, 224-233.

Gross, B., Montgomery-Brown, J., Naumann, A., Reinhard, M., 2004. Occurrence and fate of pharmaceuticals and alkylphenol ethoxylate metabolites in an effluentdominated river and wetland. Environmental Toxicology and Chemistry 23, 2074-2083.

Heberer, T., 2002. Occurrence, fate, and removal of pharmaceutical residues in the aquatic environment: a review of recent research data. Toxicology Letters 131, 5-17.

Hirsch, R., Ternes, T., Haberer, K., Kratz, K.L., 1999. Occurrence of antibiotics in the aquatic environment. Science of the Total Environment 225, 109-118.

Jones, O.A.H., Voulvoulis, N., Lester, J.N., 2002. Aquatic environmental assessment of the top 25 English prescription pharmaceuticals. Water Research 36, 50135022 .
Kim, S.D., Cho, J., Kim, I.S., Vanderford, B.J., Snyder, S.A., 2007. Occurrence and removal of pharmaceuticals and endocrine disruptors in South Korean surface, drinking, and waste waters. Water Research 41, 1013-1021.

Kolpin, D.W., Furlong, E.T., Meyer, M.T., Thurman, E.M., Zaugg, S.D., Barber, L.B., Buxton, H.T., 2002. Pharmaceuticals, hormones, and other organic wastewater contaminants in US streams, 1999-2000: a national reconnaissance. Environmental Science \& Technology 36, 1202-1211.

Kuehne, M., Ihnen, D., Moeller, G., Agthe, O., 2000. Stability of tetracycline in water and liquid manure. Journal of Veterinary Medicine Series A 47, 379-384.

Kummerer, K., Henninger, A., 2003. Promoting resistance by the emission of antibiotics from hospitals and households into effluent. Clinical Microbiology and Infection 9, 1203-1214.

Lin, A.Y.C., Reinhard, M., 2005. Photodegradation of common environmental pharmaceuticals and estrogens in river water. Environmental Toxicology and Chemistry 24, 1303-1309.

Lin, A.Y.C., Plumlee, M.H., Reinhard, M., 2006. Natural attenuation of pharmaceuticals and alkylphenol polyethoxylate metabolites during river transport: Photochemical and biological transformation. Environmental Toxicology and Chemistry 25, 1458-1464.

Loraine, G.A., Pettigrove, M.E., 2006. Seasonal variations in concentrations of pharmaceuticals and personal care products in drinking water and reclaimed wastewater in Southern California. Environmental Science \& Technology 40, 687-695.

Managaki, S., Murata, A., Takada, H., Tuyen, B.C., Chiem, N.H., 2007. Distribution of macrolides, sulfonamides, and trimethoprim in tropical waters: ubiquitous occurrence of veterinary antibiotics in the Mekong Delta. Environmental Science \& Technology 41, 8004-8010.

Miao, X.S., Bishay, F., Chen, M., Metcalfe, C.D., 2004. Occurrence of antimicrobials in the final effluents of wastewater treatment plants in Canada. Environmental Science \& Technology 38, 3533-3541.

Morrall, D., McAvoy, D., Schatowitz, B., Inauen, J., Jacob, M., Hauk, A., Eckhoff, W., 2004. A field study of triclosan loss rates in river water (Cibolo Creek, TX). Chemosphere 54, 653-660.

National Antibiotics Usage Survey Project Department of Health, 2006. Executive Yuan, Taiwan.

National Research Health Research Database, 2008d. http://www.nhri.org.tw/nhird/ index.htm (accessed April 2008)

Quanrud, D.M., Quast, K., Conroy, O., Karpiscak, M.M., Gerba, C.P., Lansey, K.E., Ela, W.P., Arnold, R.G., 2004. Estrogenic activity and volume fraction of waste water origin in monitoring wells along the Santa Cruz River, Arizona. Ground Water Monitoring and Remediation 24, 86-93.

Quinn, B., Gagne, F., Blaise, C., 2008. An investigation into the acute and chronic toxicity of eleven pharmaceuticals (and their solvents) found in wastewater effluent on the cnidarian, Hydra attenuata. Science of the Total Environment 389, 306-314

Sacher, F., Lang, F.T., Brauch, H.J., Blankenhorn, I., 2001. Pharmaceuticals in groundwaters - analytical methods and results of a monitoring program in Baden-Wurttemberg, Germany. Journal of Chromatography A 938, 199-210.

Sassman, S.A., Lee, L.S., 2005. Sorption of three tetracyclines by several soils: Assessing the role of $\mathrm{pH}$ and cation exchange. Environmental Science \& Technology 39, 7452-7459.

Ternes, T.A., Meisenheimer, M., McDowell, D., Sacher, F., Brauch, H.J., Gulde, B.H., Preuss, G., Wilme, U., Seibert, N.Z., 2002. Removal of pharmaceuticals during drinking water treatment. Environmental Science \& Technology 36, 38553863.

Tixier, C., Singer, H.P., Canonica, S., Muller, S.R., 2002. Phototransformation of triclosan in surface waters: a relevant elimination process for this widely used biocide - laboratory studies, field measurements, and modeling. Environmental Science \& Technology 36, 3482-3489.

Xu, W.H., Zhang, G., Li, X.D., Zou, S.C., Li, P., Hu, Z.H., Li, J., 2007. Occurrence and elimination of antibiotics at four sewage treatment plants in the Pearl River Delta (PRD), South China. Water Research 41, 4526-4534. 